\title{
Editorials
}

\section{Is there a place for point-of-care ultrasound in UK primary care?}

\section{INTRODUCTION}

Point-of-care ultrasound (PoC-US), defined as an ultrasound examination performed and interpreted in real time during a patient's consultation, has been described as the stethoscope of the future." Portable ultrasound devices are becoming smaller, more sensitive, and more affordable, with handheld options costing only a few thousand pounds. As a result, interest in PoC-US as an add-on to the traditional clinical assessment of patients is growing, ${ }^{2}$ and basic training is now included in the undergraduate medical curriculum of some UK universities. This leads to the question: could PoC-US have a role in UK primary care, alongside the traditional clinical examination and symptom appraisal?

\section{OPPORTUNITIES AND CHALLENGES}

The introduction of PoC-US in general practice could improve diagnostic pathways, leading to prompter referral and(or) treatment, and thereby contributing to better patient outcomes.

However, its implementation faces many challenges. First, GPs would need enough training and exposure to ensure reliable identification, especially for rarer conditions. As PoC-US is highly user dependent, a quality assurance framework is necessary to ensure both the technical skills and appropriate interpretation and integration into clinical care. Second, workload pressures may make it difficult for primary care clinicians to offer an additional service. Furthermore, increased imaging may generate harms from incidental findings and overdiagnosis, and should be integrated into a quality development scheme to monitor and minimise these potential risks.

Finally, there is little research on the use of PoC-US in family medicine, paucity of evidence-based guidelines to underpin appropriate use, and no consensus on the types of applications best suited to primary care. A recent systematic review has identified 51 studies from 18 different countries, but only 18 of these were less than 10 years old. ${ }^{3}$

\section{"The introduction of point-of-care ultrasound in general practice could improve diagnostic pathways, leading to prompter referral and(or) treatment, and thereby contributing to better patient outcomes.}

\section{LEARNING FROM ABROAD}

Despite these difficulties, there is increasing interest in family medicine PoC-US in both North America 4,5 and Europe. ${ }^{6}$ In some European countries, GPs have begun using PoC-US for the diagnosis of a number of musculoskeletal/joint, abdominal, urogenital, cardiac and vessel conditions, and for basic obstetric examinations. ${ }^{6}$ Direct in-house access to ultrasound, either performed by the primary care physician or a specialised colleague ffor example, sonographer or radiographer), is particularly common in Germany, Slovenia, and Switzerland.?

To support PoC-US implementation, several countries are developing GP-tailored educational programmes, covering the specific scans more relevant to primary care. ${ }^{4.8}$ After training is completed, periodic re-certification and performing a minimum number of scans per year are compulsory in some countries in order to maintain standards and for quality assurance. ${ }^{6}$ In the UK, availability of funding and time for both training and re-certification is a significant barrier to the adoption of PoC-US. However, studies have shown that proficiency in single, focused examinations is reached relatively quickly (2-31 hours). ${ }^{3}$ Therefore, training in low-to-moderate difficulty scans could be provided to UK GPs in a similar manner to other optional short continued professional development courses (such as, minor surgery or musculoskeletal joint injections). To minimise the burden of training and limit their absence from the clinic, UK GPs with an interest in PoC-US could build their scan portfolio gradually, similarly to family doctors abroad, who often choose to focus on specific

\section{clear evidence is needed on what type of GP scans are time- and cost-effective, and have overall benefits.}

examinations within areas of interest ${ }^{9}$

The extra capacity necessary to fit scans within GP consultations would be another major challenge for PoC-US implementation in the UK; however, a key difference with the more extensive, explorative, and diagnostic scans performed by radiologists is that GPs scans tend to be focused to answer specific and simple clinical questions, mostly aiming to rule in a diagnosis in patients with a high pre-test probability. ${ }^{10}$ Such focused examinations are quick to perform, taking an average of 5-10 minutes. ${ }^{3}$ Evidence from other primary care systems with similar time constraints suggests that GPs perform only a few scans per day, fitting them relatively easily into their normal schedule, ${ }^{6}$ although it may occasionally cause a delay. ${ }^{9}$ Nevertheless, to justify the time and financial investment required to implement PoC-US in UK primary care, clear evidence is needed on what type of GP scans are time- and cost-effective, and have overall benefits. Clinical guidelines could be used to guide PoC-US application and choice of investigations, as in other European countries.

UK GPs may also be concerned about liability in case of misdiagnosis following a scan; however, there is some evidence that the diagnostic accuracy of GPs' scans is often comparable with those performed by specialists. ${ }^{11,12}$ Additionally, a GP scan is not seen as a substitute for a specialist examination, but rather as a complement to the more traditional clinical approach. Hence, the most appropriate comparison would be between the diagnostic accuracy of physical examination alone or with the addition of PoC-US, rather than between imaging performed by GPs and specialists. Reassuringly, research also shows that the rate of false-negative and overlooked incidental findings for focused PoC-US examinations in primary care is low. ${ }^{3}$

Despite the many challenges, there is some evidence that when PoC-US is used in primary care the outcomes are positive. 


\section{“... a GP scan is not seen as a substitute for a specialist examination, but rather as a complement to the more traditional clinical approach.}

GPs have more confidence in their diagnosis, while patients report increased levels of satisfaction and reassurance. ${ }^{3}$ Earlier studies also suggest reduced referrals and overall cost-savings, ${ }^{13,14}$ but comprehensive and recent health economic evaluation is lacking.

\section{A TOOL FOR EARLIER CANCER DIAGNOSIS?}

Currently, PoC-US is not specifically used to diagnose cancer, but cancer may be suspected following a GP scan. Therefore, an early cancer diagnosis may indirectly result from PoC-US implementation for other conditions. Perhaps PoC-US could find specific niche applications in cancer diagnosis alongside some of the non-cancer roles, but more evidence is required to establish which types of applications are suited for primary care.

In future, PoC-US implementation could be facilitated by diagnostic support based on artificial intelligence, and by the ability to take and transmit ultrasound images and movies for review by specialists. For example, delay and misdiagnosis of soft-tissue sarcoma as a more benign condition is not rare in primary care. ${ }^{15}$ It is possible that remote ultrasound interpretation could streamline the assessment of patients with subcutaneous lumps in primary care, in a similar way to novel pathways for teledermatology. ${ }^{16}$ However, more research is needed to determine if such niche applications are relevant, safe, and lead to an overall improved patient outcome.

\section{CONCLUSIONS}

The experience of PoC-US in other clinical fields and primary care systems suggests a role for supporting diagnosis for some conditions. The emergence of a new generation of clinicians who have become familiar with the technology in training may herald a more widespread adoption in the future. A comprehensive programme of research is required to evaluate whether this technology can be implemented in UK primary care, followed by the development of evidence-based guidelines to support and underpin appropriate use. Whether PoC-US has a role in some well-defined aspects of cancer diagnosis remains to be established.

\section{Erica di Martino,}

Postdoctoral Research Fellow, Division of Primary Care, Public Health and Palliative Care, Leeds Institute of Health Sciences, University of Leeds, Leeds, UK.

\section{Camilla Aakjær Andersen,}

Postdoctoral Researcher, Center for General

Practice, Aalborg University, Aalborg East, Denmark.

\section{Troels Mengel-Jørgensen,}

GP and Chairman of Danish Society of Ultrasound in General Practice, Center for General Practice, Aalborg University, Aalborg East, Denmark.

\section{Martin Bach Jensen,}

GP and Professor of General Practice, Center for General Practice, Aalborg University, Aalborg East, Denmark.

\section{Stephen Bradley,}

GP and Clinical Research Fellow, Division of Primary Care, Public Health and Palliative Care, Leeds

\section{REFERENCES}

1. Filly RA. Ultrasound: the stethoscope of the future, alas. Radiology 1988; 167(2): 400.

2. Diprose W, Verster F, Schauer C. Re-examining physical findings with point-of-care ultrasound: a narrative review. N Z Med J 2017; 130(1449): 46-51.

3. Andersen CA, Holden S, Vela J, et al. Pointof-care ultrasound in general practice: a systematic review. Ann Fam Med 2019; 17(1): 61-69.

4. Hall JWW, Holman H, Bornemann P, et al. Point of care ultrasound in family medicine residency programs: a CERA study. Fam Med 2015; 47(9): 706-711.

5. Micks T, Braganza D, Peng S, et al. Canadian national survey of point-of-care ultrasound training in family medicine residency programs.

6. Mengel-Jørgensen T, Jensen MB. Variation in the use of point-of-care ultrasound in general practice in various European countries. Results of a survey among experts. Eur $J$ Gen Pract 2016; 22(4): 274-277.

7. Andersen CA, Jensen MB, Toftegaard BS, et al. Primary care physicians access to in-house ultrasound examinations across Europe: a questionnaire study. BMJ Open 2019; 9(9): e030958.

8. Løkkegaard T, Todsen T, Nayahangan LJ, et al. Point-of-care ultrasound for general practitioners: a systematic needs assessment. Scand J Prim Health Care 2020; 38(1): 3-11. Can Fam Physician 2018; 64(10): e462-e467.

\section{ADDRESS FOR CORRESPONDENCE}

\section{Erica di Martino}

Division of Primary Care, Public Health and

Palliative Care, Leeds Institute of Health Sciences, University of Leeds, Worsley Building, Level 10, Clarendon Way, Leeds LS2 9NL, UK.

\section{Email: e.dimartinoßleeds.ac.uk}

Institute of Health Sciences, University of Leeds, Leeds, UK.

\section{Richard D Neal,}

GP and Professor of Primary Care Oncology, Division of Primary Care, Public Health and Palliative Care, Leeds Institute of Health Sciences, University of Leeds, Leeds, UK.

\section{Funding}

This work arises from the CanTest Collaborative, which is funded by Cancer Research UK (ref: C8640/ A23385), of which Erica di Martino is a Postdoctoral Researcher, Stephen Bradley is a Clinical Research Fellow, and Richard D Neal is an Associate Director.

\section{Provenance}

Freely submitted; externally peer reviewed.

\section{Competing interests}

The authors have declared no competing interests.

DOI: https://doi.org/10.3399/bjgp20X710801

9. Andersen CA, Davidsen AS, Brodersen J, et al. Danish general practitioners have found their own way of using point-of-care ultrasonography in primary care: a qualitative study. BMC Fam Pract 2019; 20(1): 89

10. Bornemann P. Barreto T. Point-of-care ultrasonography in family medicine. Am Fam Physician 2018; 98(4): 200-202.

11. Lindgaard K, Riisgaard L. Validation of ultrasound examinations performed by general practitioners. Scand J Prim Health Care 2017; 35(3): 256-261.

12. Mumoli N, Vitale J, Giorgi-Pierfranceschi M et al. PRACTICUS Study Investigators. General practitioner-performed compression ultrasonography for diagnosis of deep vein thrombosis of the leg: a multicenter, prospective cohort study. Ann Fam Med 2017; 15(6): 535-539

13. Wordsworth S, Scott A. Ultrasound scanning by general practitioners: is it worthwhile? J Public Health Med 2002; 24(2): 88-94.

14. Colli A, Prati D, Fraquelli M, et al. The use of a pocket-sized ultrasound device improves physical examination: results of an in- and outpatient cohort study. PLOS One 2015; 10(3): e0122181.

15. Clark MA, Thomas JM. Delay in referral to a specialist soft-tissue sarcoma unit. Eur J Surg Oncol 2005; 31(4): 443-448

16. Lee JJ, English JC, 3rd. Teledermatology: a review and update. Am J Clin Dermatol 2018; 19(2): 253-260 